\title{
A temporal gene expression map of Chrysanthemum leaves infected with Alternaria alternata reveals different stages of defense mechanisms
}

\author{
Ye Liu', Jingjing Xin ${ }^{1}$, Lina Liu', Aiping Song ${ }^{1}$, Zhiyong Guan ${ }^{1}$, Weimin Fang ${ }^{1}$ and Fadi Chen ${ }^{1}$
}

\begin{abstract}
Chrysanthemum (Chrysanthemum morifolium) black spot disease (CBS) poses a major threat to Chrysanthemum cultivation owing to suitable climate conditions and current lack of resistant cultivars for greenhouse cultivation. In this study, we identified a number of genes that respond to Alternaria alternata infection in resistant and susceptible Chrysanthemum cultivars. Based on RNA sequencing technology and a weighted gene coexpression network analysis (WGCNA), we constructed a model to elucidate the response of Chrysanthemum leaves to A. alternata infection at different stages and compared the mapped response of the resistant cultivar 'Jinba' to that of the susceptible cultivar 'Zaoyihong'. In the early stage of infection, when lesions had not yet formed, abscisic acid (ABA), salicylic acid (SA) and EDS1-mediated resistance played important roles in the Chrysanthemum defense system. With the formation of necrotic lesions, ethylene (ET) metabolism and the $\mathrm{Ca}^{2+}$ signal transduction pathway strongly responded to $A$. alternata infection. During the late stage, when necrotic lesions continued to expand, members of the multidrug and toxic compound extrusion (MATE) gene family were highly expressed, and their products may be involved in defense against $A$. alternata invasion by exporting toxins produced by the pathogen, which plays important roles in the pathogenicity of $A$. alternata. Furthermore, the function of hub genes was verified by qPCR and transgenic assays. The identification of hub genes at different stages, the comparison of hub genes between the two cultivars and the highly expressed genes in the resistant cultivar 'Jinba' provide a theoretical basis for breeding cultivars resistant to CBS.
\end{abstract}

\section{Introduction}

The production of cut Chrysanthemum flowers growing under greenhouse conditions is threatened by many diseases. Alternaria alternata, a common necrotrophic fungus that is ubiquitously present on various plant species, damages plant tissues by producing toxins ${ }^{1,2}$. Warm and humid environmental conditions lead to the spread of diseases caused by this fungus, which is a year-round problem for Chrysanthemum production in greenhouses.

\footnotetext{
Correspondence: Fadi Chen (chenfd@njau.edu.cn)

${ }^{1}$ State Key Laboratory of Crop Genetics and Germplasm Enhancement, Key Laboratory of Landscaping, Ministry of Agriculture and Rural Affairs, College of Horticulture, Nanjing Agricultural University, Nanjing, China

These authors contributed equally: Ye Liu, Jingjing Xin
}

Although the early stage of the infection is symptomless, small round black spots form at the site of A. alternata invasion, and these spots eventually expand into round, round-like, or irregular spots that are covered with a dark mildew layer. The widespread occurrence of this disease severely affects the yield and ornamental value of Chrysanthemum, causing substantial losses in flower production.

Currently, Chrysanthemum black spot disease (CBS) is controlled by fungicides; however, residues and severe environmental pollution caused by fungicides contradict the green, sustainable development of agricultural production. Therefore, screening and breeding resistant Chrysanthemum cultivars is the most environmentally

\section{(c) The Author(s) 2020}

(c) (i) Open Access This article is licensed under a Creative Commons Attribution 4.0 International License, which permits use, sharing, adaptation, distribution and reproduction cc) in any medium or format, as long as you give appropriate credit to the original author(s) and the source, provide a link to the Creative Commons licence, and indicate if changes were made. The images or other third party material in this article are included in the article's Creative Commons licence, unless indicated otherwise in a credit line to the material. If material is not included in the article's Creative Commons licence and your intended use is not permitted by statutory regulation or exceeds the permitted use, you will need to obtain permission directly from the copyright holder. To view a copy of this licence, visit http://creativecommons.org/licenses/by/4.0/. 
friendly approach to controlling CBS. Furthermore, revealing the molecular mechanism of CBS resistance will provide a strong theoretical basis for formulating disease resistance breeding strategies and preventing black spot disease. In our laboratory, a variety of Chrysanthemum germplasm resources with different levels of resistance to black spot disease are being used to identify key genes involved in disease resistance.

The response of the plant immune system to pathogen infection involves two branches: PTI (pathogen-associated molecular pattern-triggered immunity) and ETI (effectortriggered immunity $)^{3}$. PTI is mainly triggered by the perception of microbial proteins, leading to the deposition of callose in the cell wall, MAP kinase cascade signal transduction, changes in gene expression, closure of stomata, and/or an oxidative burst ${ }^{4-6}$. ETI is regulated by resistance genes ( $R$ genes). Activation of $R$ genes leads to reactive oxygen production and the hypersensitive response (HR), which is considered a programmed cell death (PCD) response to impede expansion of the pathogen ${ }^{7-9}$. During the interaction between plants and pathogens, $R$ genes play significant roles in the HR; the SA-dependent defense signaling pathway is invoked when the effector is recognized by an $R$ gene ${ }^{10,11}$. SA signaling is also an important aspect of the PAMP response and $R$ gene-mediated resistance ${ }^{12,13}$. Both ET and jasmonic acid, which are plant hormones, are crucial for the $\mathrm{PCD}$ response $^{14-17}$. Additionally, abscisic acid (ABA) plays a positive role in early-acting defenses, such as stomatal closure, but plays a negative role in later-acting defense mechanisms mediated by SA or $\mathrm{JA}^{18-20}$. These immune responses are controlled by the reprogramming of a considerable number of defense-related genes, including those encoding transcription factors (TFs) $)^{21,22}$.

The plant immune response is closely related to the calcium ion $\left(\mathrm{Ca}^{2+}\right)$ signal transduction pathway via the decoding of the $\mathrm{Ca}^{2+}$ signatures triggered by pathogens ${ }^{23-27}$. Different $\mathrm{Ca}^{2+}$ sensors have been reported to recognize $\mathrm{Ca}^{2+}$ signatures, such as calmodulin-like proteins (CMLs) and calmodulin $(\mathrm{CaM})$, demonstrating that the conversion of $\mathrm{Ca}^{2+}$ signals to plant immune responses is mediated by different channels and machineries ${ }^{28}$. As a general secondary messenger, $\mathrm{Ca}^{2+}$ participates in different types of cellular processes and has important functions in sensing microbe signals to establish appropriate immune and symbiotic responses, including the production of ROS and $\mathrm{NO}$ as well as $\mathrm{PCD}^{26}$. Moreover, $\mathrm{Ca}^{2+}$ signaling is connected to other hormone-mediated pathways. In guard cells, stomatal closure in response to increased levels of $\mathrm{Ca}^{2+}$ is impaired in $c p k 5 / 6 / 11 / 23$ mutants treated with $\mathrm{ABA}^{29}$. Research also shows that $\mathrm{Ca}^{2+}$ is involved in the ET-mediated pathogenesis response ${ }^{30}$. Moreover, many TFs have been identified as being involved in calcium signaling. One class of TFs comprising $\mathrm{Ca}^{2+} / \mathrm{CaM}-$ regulated members is the WRKY family; WRKY43, WRKY53, and all members of the WRKYIId subfamily have been found to interact with $\mathrm{Ca}^{2+} / \mathrm{CaM}^{31}$. Although the $\mathrm{Ca}^{2+}$ signal transduction pathway has been a popular research topic for many years, it remains unknown whether $\mathrm{Ca}^{2+}$ is involved in Chrysanthemum-A. alternata interaction.

RNA sequencing technology is an efficient method for revealing genetic diversity and comparing variation in global expression of coding genes among genotypes/cultivars. Several transcriptome studies involving various genotypes/cultivars have been performed to understand stress responses ${ }^{32}$. Nevertheless, no such detailed analysis has been performed to elucidate the molecular regulatory mechanisms underlying the response of both resistant and susceptible Chrysanthemum cultivars to A. alternata infection.

Here, we dissect RNA sequencing time series data to reveal the transcriptional regulatory network in Chrysanthemum leaves after A. alternata inoculation. Our integrated analysis revealed that EDS1-mediated disease resistance and hormone and $\mathrm{Ca}^{2+}$ signal transduction pathways are of vital importance in the response of Chrysanthemum to infection of this fungus. Differentially expressed gene (DEG) and coexpression network analyses revealed a series of hub genes that play key roles at different stages of $A$. alternata infection. To verify the function of these hub genes, we performed transgenic experiments with CmWRKY33.1 and acquired senseexpression genetic transformation lines. Inoculation assays showed that three lines overexpressing CmWRKY33.1 exhibited enhanced susceptibility to black spot disease compared with that of wild-type 'Jinba'. Our research presents insight into the molecular regulatory mechanisms underlying Chrysanthemum responses to $A$. alternata inoculation.

\section{Results \\ Evaluation of Chrysanthemum cultivars after inoculation with $A$. alternata}

We selected two Chrysanthemum cultivars, 'Jinba' (resistant cultivar) and 'Zaoyihong' (susceptible cultivar), which differ significantly in their symptoms after $A$. alternata inoculation. The black leaf spot disease symptoms of the two Chrysanthemum cultivars were investigated at 3 days post inoculation. 'Jinba (SM)' displayed necrotic lesions that were mostly limited to the inoculated leaf, whereas 'Zaoyihong (ZYH)' exhibited necrotic lesions on other leaves, including the leaf inoculated with $A$. alternata (Fig. 1a). The disease symptoms were evaluated based on the proportion of diseased leaves at 3 days post inoculation; as shown in Fig. 1b, the proportions of diseased 'Jinba' and 'Zaoyihong' leaves were $0.08-0.10$ and $0.45-0.56$, respectively. 
a

Jinba
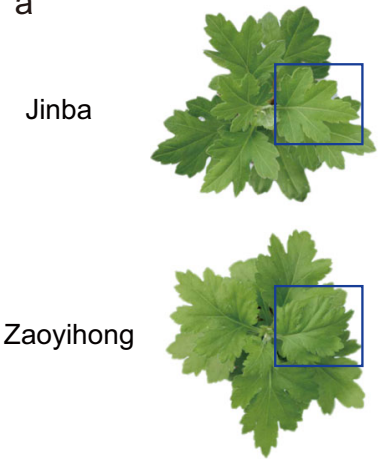

Control
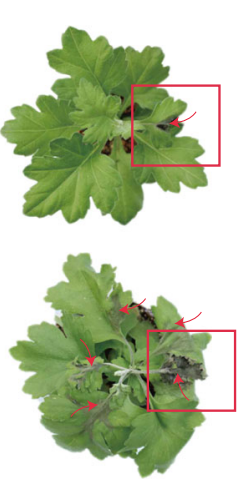

Infection

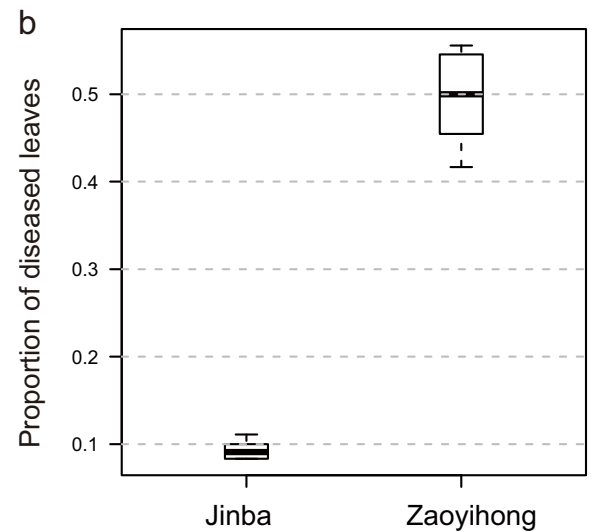

Fig. 1 Evaluation of the response of two Chrysanthemum cultivars to Chrysanthemum black spot disease (CBS). a Images of the resistant cultivar 'Jinba' and the susceptible cultivar 'Zaoyihong' with CBS. b Proportion of diseased leaves of A. alternata infections in 'Jinba' and 'Zaoyihong'

Leaves inoculated with $A$. alternata were sampled at six time points $(0 \mathrm{~h}, 1 \mathrm{~h}, 6 \mathrm{~h}, 12 \mathrm{~h}, 24 \mathrm{~h}$, and $36 \mathrm{~h})$ based on the phenotypes to analyze differences in transcript levels between 'Jinba' and 'Zaoyihong', with three biological replicates sampled for each cultivar at each time point.

\section{Generation of transcriptomic time series data via RNA sequencing}

RNA sequencing technology is an effective method for revealing genetic diversity and comparing the variation in global expression of coding genes among various genotypes/cultivars. To further investigate the transcriptome dynamics and regulatory mechanism of Chrysanthemum leaf responses to $A$. alternata, we performed a comparative transcriptome-wide time series analysis of the two Chrysanthemum cultivars, 'Zaoyihong (ZYH)' and 'Jinba (SM)', with three independent biological replicates for all samples at each time point. In total, $36 \mathrm{cDNA}$ libraries from the leaf tissues of the two cultivars were generated: SM_0_1, SM_0_2, SM_0_3, SM_1_1, SM_1_2, SM_1_3, SM_6_1, SM_6_2, SM_6_3, SM_12_1, SM_12_2, SM_12_3, SM_24_1, SM_24_2, SM_24_3, SM_36_1, SM_36_2, SM_36_3, ZYH_0_1, ZYH_0_2, ZYH_0_3, ZYH_1_1, ZYH_1_2, ZYH_1_3, ZYH_6_1, ZYH_6_2, ZYH_6_3, ZYH_12_1, ZYH_12_2, ZYH_12_3, ZYH_24_1, ZYH_24_2, $\mathrm{ZYH} \_24 \_3, \mathrm{ZYH}$ 36_1, ZYH_36_2, and ZYH_36_3. The raw data were then converted to clean reads, which were subsequently used for further analysis (Table S1). As a result, 1.7 billion clean reads (average of $\sim 23.3$ million reads from each sample) were obtained. All clean data can be downloaded from NCBI (BioProjects: PRJNA596929).

\section{Analysis of DEGs in the time series samples}

RSEM and BOWTIE were used to process the clean reads for each sample to obtain the expected FPKM (fragments per kilobase of transcript length per million mapped reads) number for each gene. The DESeq $R$ package was used to identify differentially expressed genes. Pearson correlation analysis was then applied to compare the RNA sequencing data of the three biological replicates, which varied from 0.87 to 0.93 , indicating high consistency among the replicates (Fig. S1).

To investigate the temporal dynamics of transcriptional reprogramming between the two Chrysanthemum cultivars following challenge with $A$. alternata, we plotted the number of DEGs (padj <0.05) against those of noninoculated plants (SM_0 or ZYH_0) at each inoculation time point. In the 'Jinba' cultivar, 5740 (2835 upregulated; 2905 downregulated), 19,691 (12,176 upregulated; 7515 downregulated), 13,805 (8868 upregulated; 4937 downregulated), 25,549 (16,315 upregulated; 9234 downregulated $)$ and 25,375 (15,543 upregulated; 9732 downregulated) DEGs were found in the samples at the five inoculation times; 7104 (3352 upregulated; 3752 downregulated), 12,010 (7129 upregulated; 4881 downregulated), 17,662 (11,277 upregulated; 6385 downregulated), 9813 (7172 upregulated; 2641 downregulated) and 18,826 (11,816 upregulated; 7010 downregulated) DEGs were identified in 'Zaoyihong' (Fig. 2a). The largest number of stage-specific genes (25,549 in 'Jinba' and 18,826 in 'Zaoyihong') was identified in the comparison of SM_24 and ZYH_36 compared with that of SM_0 and ZYH_0 (Fig. 2a). In total, 39,802 DEGs were obtained from the two cultivars; of these, 2084 DEGs were shared among the five time series stages in 'Jinba', and 3144 DEGs were shared in 'Zaoyihong'. We also identified DEGs uniquely expressed at each time series stage, as shown in Fig. 2b, and the results suggest that each stage has its own independent defense mechanisms.

The results of our study showed that a large number of DEGs, up to 33,343 in the resistant cultivar 'Jinba' and 26,173 in the susceptible cultivar 'Zaoyihong', respond to A. alternata infection. This is a considerably greater 


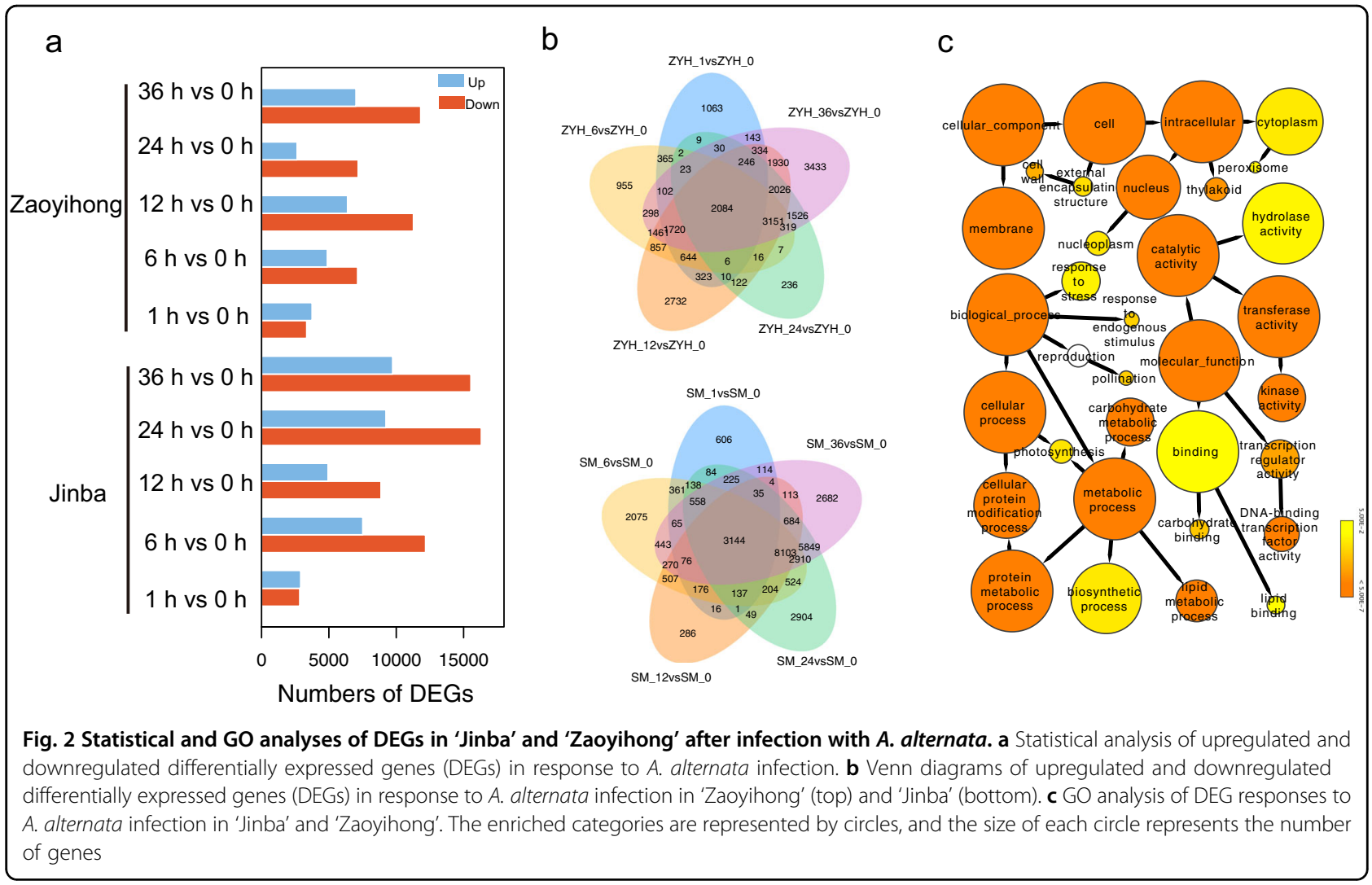

number of genes than previously reported in similar transcriptomic studies. By using BINGO, we next performed GO analysis to further characterize the identified DEGs. As shown in Fig. 2c, the DEGs were distributed among the three GO categories: "biological process", "molecular function", and "cellular component". The DEGs enriched in the "cellular component" category are mainly involved in "cell wall", "peroxisome", "nucleoplasm", and "thylakoid". Enriched terms in the "biological process" category mainly included "response to stress", "response to endogenous stimulus", and "carbohydrate metabolic process". The GO terms "hydrolase activity", "kinase activity", "DNA-binding transcription factor activity", "carbohydrate binding", and "lipid binding" were enriched in the "molecular function" category.

\section{Pathway analysis of Chrysanthemum in response to $A$. alternata infection}

To facilitate our analysis of the plant and fungal networks, we also mapped the pathways of the DEGs involved in Chrysanthemum- $A$. alternata interactions using the KEGG database (www.genome.jp/kegg). Plant hormones are crucial for helping plants adapt to adverse environmental conditions. Our data showed that the expression of genes involved in hormone signaling, especially that of ET and ABA, was upregulated when Chrysanthemum leaves were challenged with A. alternata (Fig. S2b and c); the expression of a small proportion of genes involved in other hormone signal transduction pathways was also upregulated (Fig. S3). Traditionally, it has been thought that mainly ET and JA respond to necrotrophic fungi, but SA mediates defense signaling against both hemibiotrophic and biotrophic fungi. Based on our data, SA might also play a significant role in local immunity against necrotrophic fungi (Fig. S3). Moreover, plant-pathogen interaction pathway analysis indicated that the expression of genes involved in the $\mathrm{Ca}^{2+}$-dependent signaling pathway was upregulated by more than 2 -fold (Fig. S2a).

The transcriptional changes in the leaves of resistant and susceptible Chrysanthemum cultivars infected with $A$. alternata were also visualized via MapMan software. Figure S4 depicts the general overview of the cellular response, confirming that the number of DEGs responding to biotic stress was greater than the number responding to other stresses. Figure S5 illustrates a general overview of the biotic stress response. MapMan visualization revealed that many DEGs are involved in cell wall strengthening, transcriptional regulation, the oxidative respiratory burst, cellular signaling, and hormone metabolism. Upregulated genes play important roles in the response to pathogen infection. Moreover, the expression of most of the DEGs related to ET metabolism, 
the respiratory burst and mitogen-activated protein kinases (MPKs) was upregulated at different times after $A$. alternata inoculation, which suggests the importance of these pathways for the resistance of Chrysanthemum to infection by this fungus.

In total, the involvement of multiple signal transduction pathway genes suggests that the response of Chrysanthemum leaves to A. alternata infection is not simply linear cascades but rather is a combination of multiple signaling pathways. Previous studies have shown that ET and the $\mathrm{H}_{2} \mathrm{O}_{2}$-mediated $\mathrm{HR}$ and $\mathrm{PCD}$ play vital roles in sand pear $-A$. alternata interaction ${ }^{33}$. In our study, we identified that ET, $\mathrm{ABA}$, and $\mathrm{Ca}^{2+}$ are important in the defense response to $A$. alternata inoculation and highlight the regulatory mechanism occurring in Chrysanthemum leaves.

\section{Identification of gene coexpression modules during $A$. alternata infection}

WGCNA is a powerful tool for identifying a set of genes associated with phenotypes. To investigate the gene regulatory network of Chrysanthemum leaves in response to A. alternata infection, we performed a WGCNA by using RNA sequencing profiles. After the expression data were preprocessed, a total of 36,403 genes were subjected to WGCNA, and we ultimately discovered 14 gene coexpression modules. The number of genes in these modules ranged from 62 to 8235 .

The eigengenes for the 14 modules correlated with different infection stages (Fig. 3). We studied the preservation of coexpression modules between 'Jinba' and 'Zaoyihong' at different stages of $A$. alternata infection via module-trait relationship and module-sample relationship analyses. In most of the modules, the trend of genes was nearly the same between the two cultivars at different inoculation times, as indicated by the yellow, tan, and brown colors in the figure. Interestingly, a few modules differed in response to A. alternata infection. Notably, we identified one module of 'Jinba', i.e., that associated with the turquoise color in the figure, that was cultivar specific.

\section{Transcriptional regulatory modules associated with the expansion of necrotic lesions}

We investigated the transcriptome of Chrysanthemum leaves infected with A. alternata and collected samples at six time points. The early stage of infection was symptomless up to $\sim 1 \mathrm{~h}$ post inoculation (hpi). The first signs of necrosis were observed at $6 \mathrm{hpi}$, and round or irregular lesions formed at $12 \mathrm{hpi}$ at the inoculation sites, which was considered the middle stage/period of infection. During the late stage of infection, the lesions expanded to the outside of the inoculation site at $24 \mathrm{hpi}$, and half of the Chrysanthemum leaves showed black necrotic spots at $36 \mathrm{hpi}$. Plant defense genes play crucial roles during fungal infection, and the expression of many is regulated by TFs. Therefore, we further analyzed the important defense genes and TFs with a strong regulatory relationship with the coexpressed defense genes in these notable modules.

Combined with our WGCNA, we identified key genes (also called hub genes) at different infection stages, as shown in Fig. 4. The expression of the genes in the yellow module were most upregulated at $1 \mathrm{hpi}$, corresponding to the early stage of the response of Chrysanthemum leaves to A. alternata infection. At $6-12 \mathrm{hpi}$, there was one tan module in which the gene expression showed peak upregulation at $6 \mathrm{hpi}$ or $12 \mathrm{hpi}$. Many genes with a transcriptional peak at $24 \mathrm{hpi}$ or $36 \mathrm{hpi}$ were classified into the black or brown module; at $24 \mathrm{hpi}$ or $36 \mathrm{hpi}$, the late stage, the lesions expanded outside of the inoculation site. In the yellow module, three hub genes, e.g., an EDS1 homolog (CmEDS1; DN78955co_g1), an MLO1 homolog (CmMLO1; DN86381c0_g1), and an NPR4 homo$\log$ (CmNPR4; DN80377c1_g1), related to defense were identified. Because TFs play important roles in gene regulation and plant immunity, we further investigated their regulatory network. TFs showed a strong regulatory relationship (weight/topological overlap matrix (TOM) $>0.3$ ) with other genes in the module and were used for further analysis. The identified WRKY4 homolog showed a regulatory relationship with the three hub defense response genes. In addition, the expressions of the RPS4 homolog and PYL4 homolog were upregulated in the yellow module (Fig. 4a and Fig. S2c). In the tan module, we identified two hub genes related to plantpathogen interactions: a WRKY33 homolog (CmWRKY33; DN63096c1_g1) and a CML45 homolog (CmCML45; DN69937c2_g1). An ERF2 homolog (CmERF2; DN90778 c3_g1), whose product plays important roles in the ET signal transduction pathway, was found to have a strong regulatory relationship with the two hub genes (CmWRKY33 and CmCML45) (Fig. 4b). In the late stage of the Chrysanthemum response to $A$. alternata infection, we identified two hub genes belonging to the MATE family, which are involved in multiple drug and toxic compound extrusion; a DTX30 homolog (CmDTX30; DN96458c3_g7); and a DTX32 homolog (CmDTX32; DN73836c0_g2). One TF, a WRKY6 homolog (CmWRKY6; DN87555c0_g2), was also identified as a hub gene. Furthermore, an HSF24 homolog (DN8266 2c0_g1) and a NAC029 homolog (DN73063c1_g1) exhibited strong regulatory relationships with other genes in the brown module, which also had regulatory relationships with two hub genes (CmDTX30 and CmDTX32) (Fig. 4c). These results suggest a previously unknown function for MATE family genes in response to A. alternata infection.

\section{Coexpression networks reveal that the highly resistant cultivar 'Jinba' uniquely responds to $A$. alternata infection}

The Chrysanthemum cultivar 'Jinba' was more resistant and had smaller necrotic lesions than 'Zaoyihong' did during infection with A. alternata (Fig. 5a). The 
a Network heatmap plot for selected genes
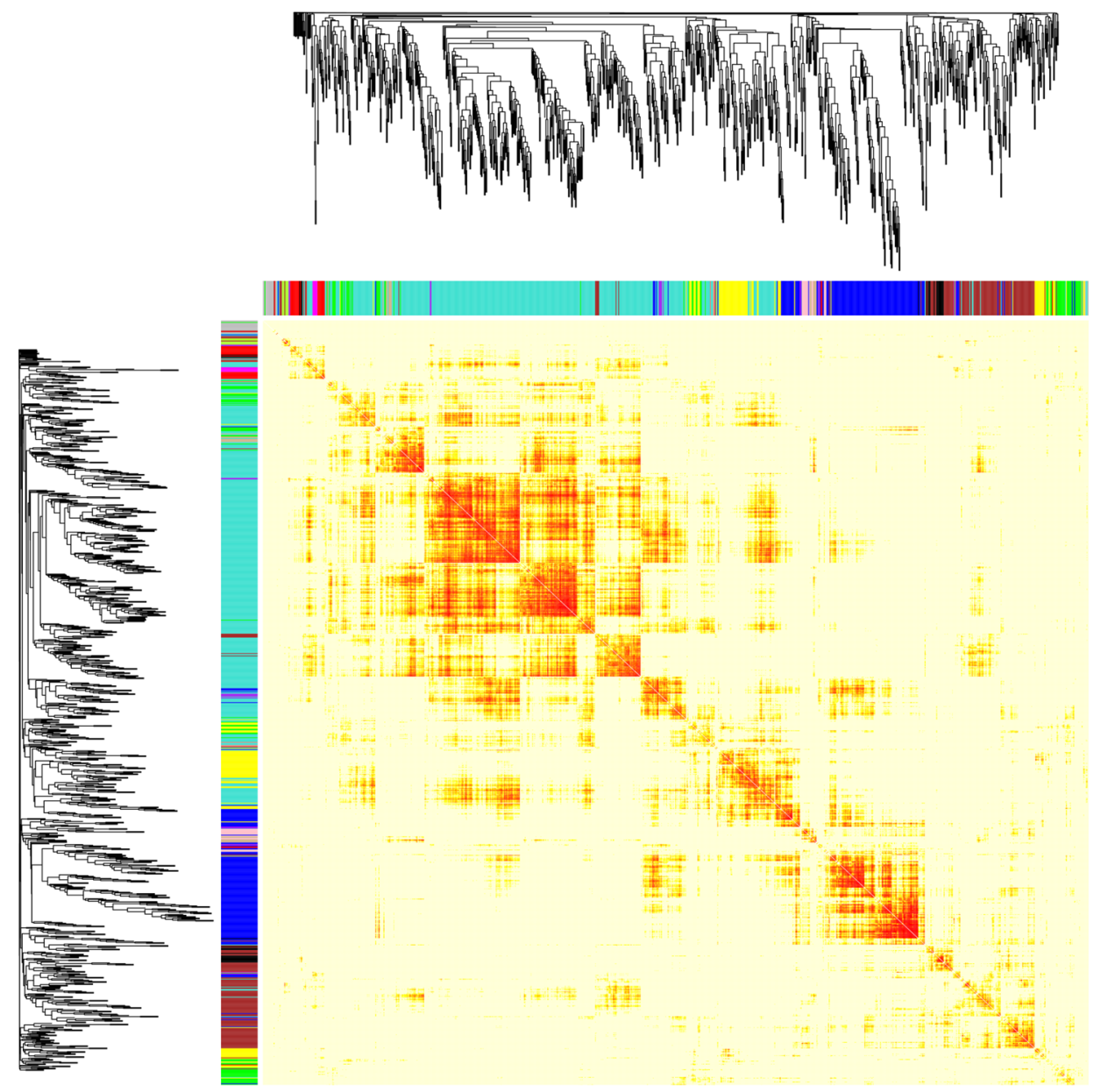

b

Module-sample relationship

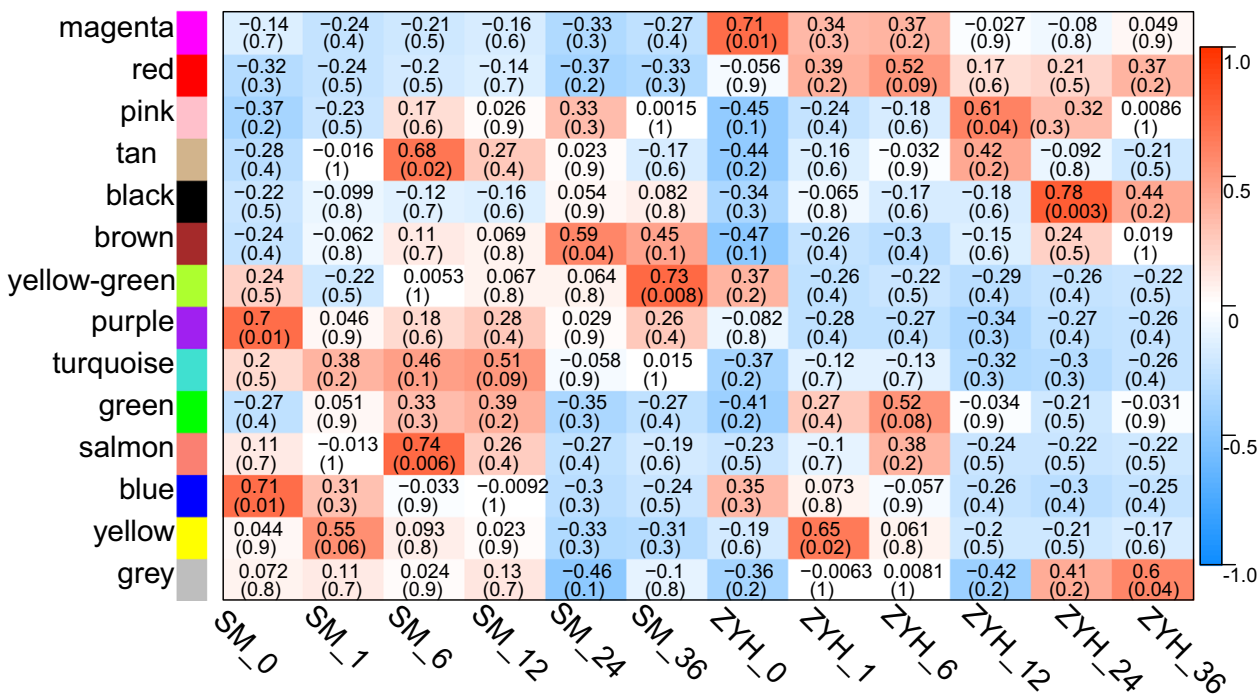

Fig. 3 WGCNA of the transcripts in 'Jinba' and 'Zaoyihong'. a Dendrogram of genes based on a coexpression network analysis in 'Jinba' and 'Zaoyihong'. Each leaf in the tree corresponds to an individual gene. Fourteen modules, each associated with a different color, formed from the major tree branches. $\mathbf{b}$ Association between modules and traits. The color of each module is the same as that in $\mathbf{a}$. Each column represents a leaf necrotic lesion trait. The legend indicates the correlation. Each cell contains the number of corresponding correlations and $P$ values 
a

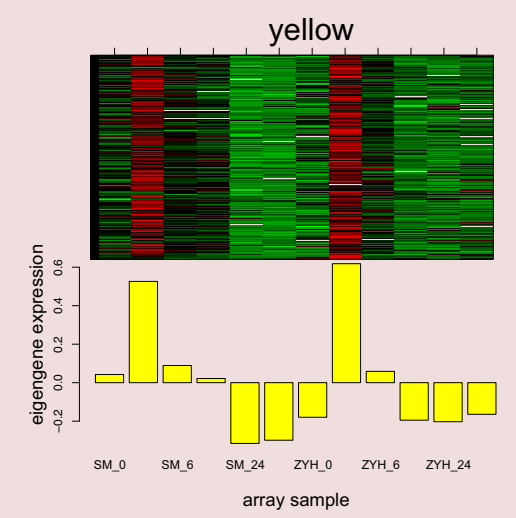

Early stage

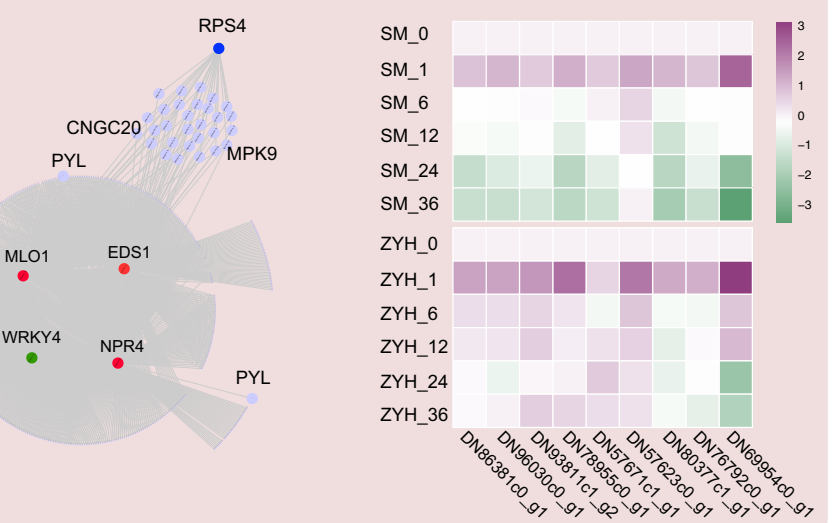

b
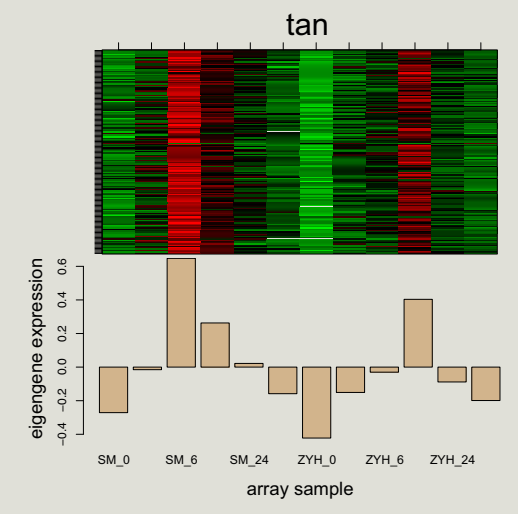

C

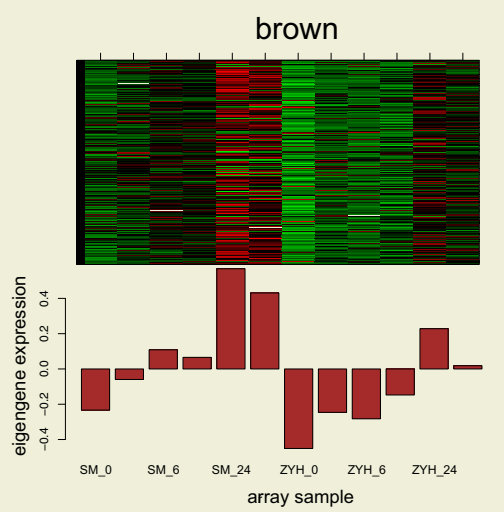

Middle stage

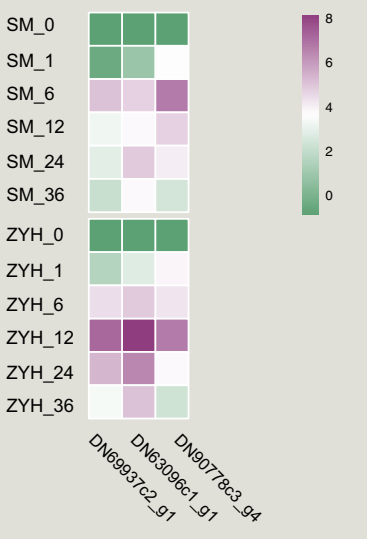

\section{Late stage}

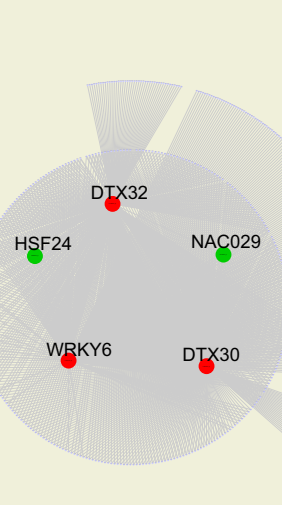

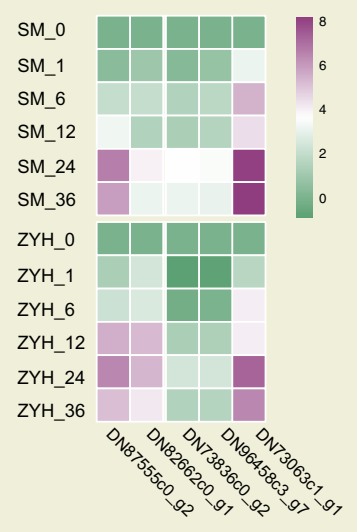

Fig. 4 (See legend on next page.) 
(see figure on previous page)

Fig. 4 DEGs at different stages of the response of Chrysanthemum leaves to A. alternata infection. a Coexpression network of the yellow module. $\mathbf{b}$ Coexpression network of the tan module. c Coexpression network of the brown module. The coexpressed genes in each module are shown in heatmaps and bar graphs (left). The red rectangles in the heatmap represent high expression; the green rectangles represent low expression. The network of top hub genes is indicated by larger circles and the red color in the network (middle). Transcription factors with strong regulatory relationships (weight $>0.3$ ) in the yellow modules are indicated by larger circles and green color in the network. The dots around the circle represent other coexpressed genes indicated by small dots, and the relationships are connected by lines. The heatmaps (right) show the expression of the top hub genes and transcription factors that present strong regulatory relationships with the top hub genes

a

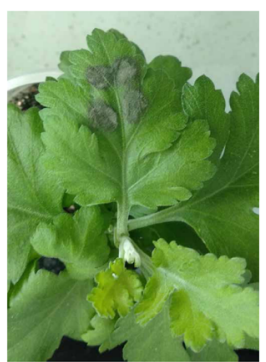

Zaoyihong

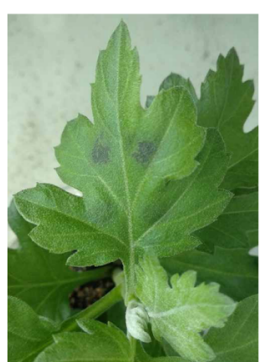

Jinba
C

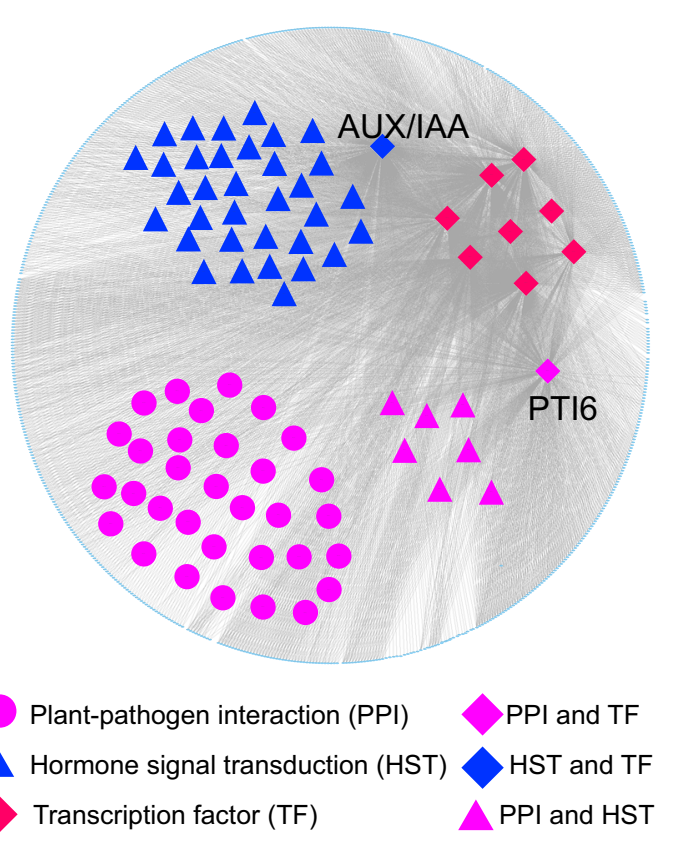

b

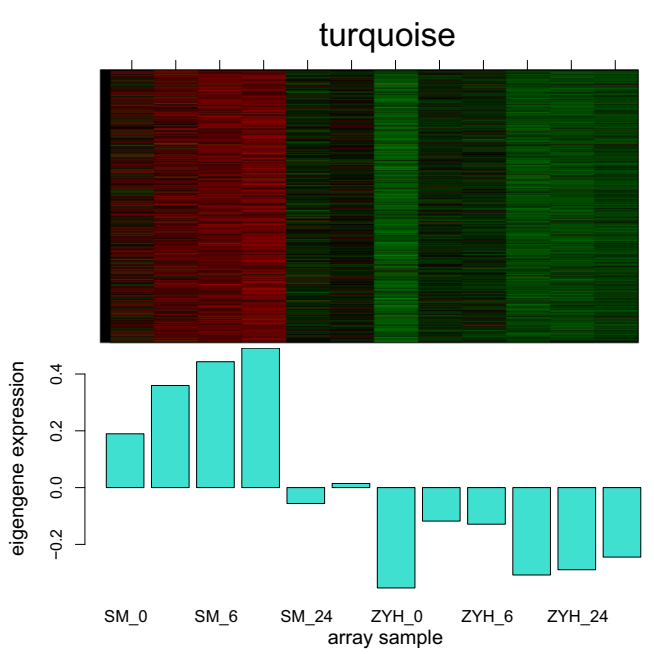

d

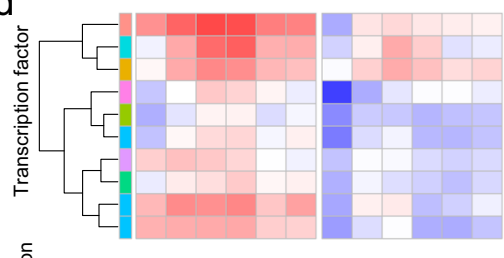

DN57265c0_g1 TF DN83617 0 g2 AP2-EREBP DN92488c0_g2 DN80856c2_g1 DN67663c1_g1 MYB DN85982c0_g1 PLATZ DN74312c3 g DN75091c0_g1 DN79798c2_g1

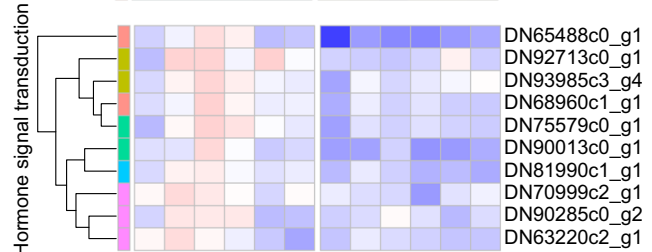
ᄃ

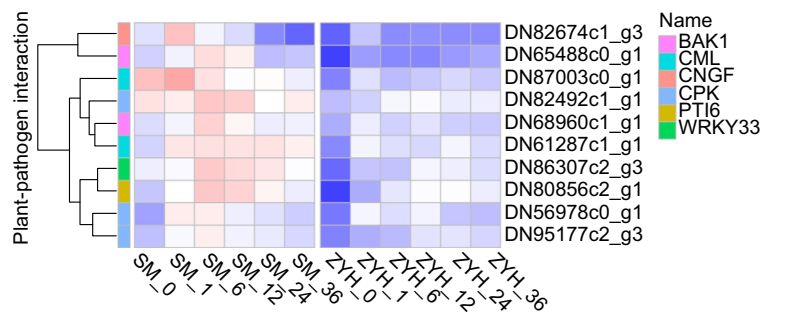

Fig. 5 Analysis of highly expressed genes in the resistant cultivar 'Jinba'. a Phenotype of Chrysanthemum leaves at $12 \mathrm{~h}$ post inoculation in 'Jinba' and 'Zaoyihong'. b The coexpressed genes are shown in heatmaps and bar graphs in the turquoise module. The red rectangles in the heatmap represent high expression; the green rectangles represent low expression. c The turquoise module was visualized by Cytoscape. Differently expressed transcription factors are indicated in larger red, blue, or purple quadrangles. Larger purple circles, triangles, or quadrangles represent differentially expressed genes involved in the plant-pathogen interaction pathway. Differentially expressed genes involved in the hormone signal transduction pathway are indicated in larger blue or purple triangles and larger blue quadrangles. $\mathbf{d}$ Heatmaps showing the expression profile of DEGs involved in PPI and HST and transcription factors in the turquoise module. The color represents the expression levels of genes in the module as shown in the figure legend 
'turquoise' module ( $r=0.83, p=9 \mathrm{e}-04)$, which included 8228 genes, was cultivar specific and upregulated after $A$. alternata infection (Fig. 5b), with a possible association with the resistant phenotype.

The genes of this module were examined for further analysis. Based on the DEG analysis, we found 10 hub genes belonging to different TF families, e.g., $A P 2-E R E B P$, $A U X, b H L H, M A D S, M Y B, N A C, P L A T Z$, and TRAF, to be differentially expressed (Fig. $5 \mathrm{~d}$ ). A total of 52 genes that interact with TFs according to the WGCNA are involved in plant-pathogen interactions, including DN80856c2_g1. Interestingly, DN80856c2_g1, called PTI6, which belongs to the AP2-EREBP TF family, also plays significant roles in plant-pathogen interactions. The results showed that the hub TFs may interact with genes involved in plant-pathogen interactions. Additionally, among the genes interacting with the hub TFs, 43 are involved in the hormone signal transduction pathway, which may be crucial in resistant cultivars challenged with A. alternata. The regulatory relationship was visualized with Cystoscope software, as depicted in Fig. 5c. Analysis of the DEGs interacting with TFs revealed one ABCA3 family gene (DN91413c1_g3) and four MATE family genes (DN94014c1_g1; DN90910c1_g1; DN95310c2_g2 and $\left.D N 82887 c 0 \_g 1\right)$ that were distinctly highly expressed in the resistant cultivar 'Jinba' (Fig. 5d and Table S3), suggesting a previously unknown function in resistance to pathogen infection.

\section{Validation of DEGs}

To verify the RNA sequencing data, we selected 10 DEGs from different modules for quantitative real-time
PCR (qPCR) assays; the housekeeping gene $E F 1 \alpha$ was used as an internal control. The DEGs included CmCML45 (DN69937c2_g1), CmWRKY33 (DN63096c1_g1), CmERF2 (DN90778c3_g1), CmNAC029 (DN73063c1_g1), CmMLO6 (DN77454c0_g1), CmMYB15 (DN77779c0_g1), CmHSF24 (DN82662c0_g1), CmDTX24 (DN81968c0_g1), CmFTSH (DN87341c1_g3), and CmVTE3 (DN93367c1_g4). The final qPCR data for the 10 DEGs were in accordance with the results of the transcriptome analysis, as presented in Fig. S6. Additionally, the qPCR and RNA sequencing results showed consistency according to a correlation analysis. The Pearson correlation coefficients of the results between the qPCR and RNA sequencing were $0.80-1$ and $0.77-1$ for the genes of 'Jinba' and 'Zaoyihong', respectively, at different stages of disease lesion development (Fig. S7), which also indicates that the data produced via RNA sequencing are reliable.

\section{Overexpression of CmWRKY33.1 resulted in enhanced susceptibility to black spot disease}

In our study, we found that WRKY family genes were highly expressed as the lesion spread. Based on the WRKY33 homologous sequences from our transcriptome data, we cloned one WRKY33 homologous gene: CmWRKY33.1. To further verify the function of CmWRKY33.1, we constructed a pMDC43-CmWRKY33.1 expression vector for genetic transformation of Chrysanthemum and acquired sense-expression transformed lines. Electrophoresis detection assays showed that the vector was successfully transformed into wild-type 'Jinba' (Fig. 6a), and according to qPCR assays, the expression of CmWRKY33.1 in the transformed lines significantly
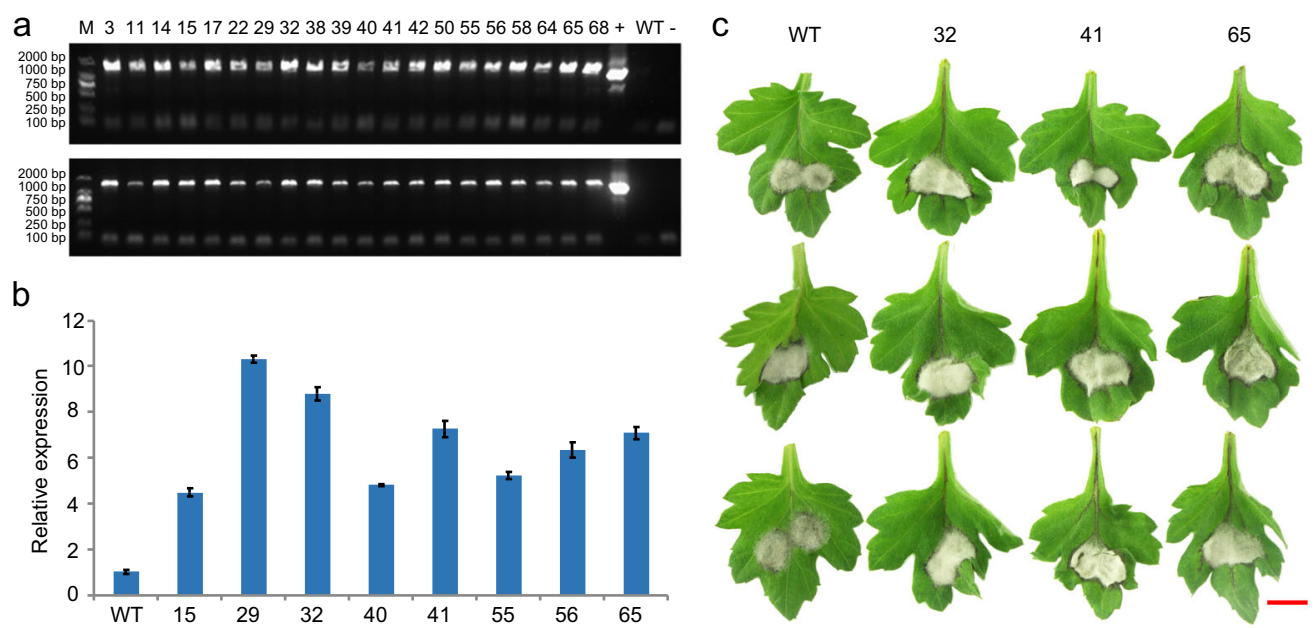

Fig. 6 Detection of CmWRKY33.1 transgenic lines and phenotypes after inoculation with A. alternata. a Electrophoresis detection of transgenic lines. 3-68: Overexpressing lines; + Positive control (Plasmid DNA), - Negative control ( $\left.\mathrm{H}_{2} \mathrm{O}\right)$, WT Wild-type 'Jinba'. Up: PCR primers CmWRKY33.1-ID-F1/R1 were used for amplification of the PMDC43-CmWRKY33.1 vector in the transformation lines. Down: PCR primers CmWRKY33.1ID-F2/R2 were used for amplification of the pMDC43-CmWRKY33.1 vector in the transformation lines. $\mathbf{b}$ Relative expression of CmWRKY33.1 in the overexpression lines. c Phenotypic differences of wild-type and CmWRKY33.1-overexpressing lines. Bar $=10 \mathrm{~mm}$ 
increased (Fig. 6b). Moreover, inoculation assays demonstrated that, compared with wild-type 'Jinba', the three transformation lines overexpressing CmWRKY33.1 displayed enhanced susceptibility to black spot disease (Fig. 6c).

\section{Discussion}

The molecular mechanisms underlying the defense system of Chrysanthemum leaves in response to biotic stress are poorly understood. In our research, we identified a series of genes that respond to A. alternata at different times after inoculation in both susceptible and resistant Chrysanthemum cultivars and aimed to elucidate the mechanism by which the resistant cultivar 'Jinba' specifically engages its defense systems. RNA sequencing was applied to detect dynamic changes in the transcriptome of two Chrysanthemum cultivars (the resistant cultivar 'Jinba' and the susceptible cultivar 'Zaoyihong') and to investigate the molecular mechanism underlying lesion expansion. Analysis of the transcriptome time series and WGCNA revealed that pathogen-induced stress triggers defense responses in Chrysanthemum. Our research thus elucidated how Chrysanthemum conveys various signals in response to $A$. alternata infection.

When no lesions formed on Chrysanthemum leaves after A. alternata inoculation, the defense regulator CmEDS1 plays significant roles in the response to infection, which is consistent with the involvement of CmRPS4, CmNPR4, and CmPYL4. In Arabidopsis, EDS1 together with RPS4 activates the TIR-NB-LRR signal transducer for defense across cellular compartments ${ }^{34-36}$. Moreover, EDS1 is an upstream regulator of the SA signaling pathway, and the NPR4 gene codes for a protein that acts as an SA receptor ${ }^{37,38}$. Our results showed that the EDS1-, ABA-, and SA-mediated disease resistance pathways in Chrysanthemum have crucial roles in the early response to A. alternata infection. In Arabidopsis, WRKY4 acts as a positive regulator of plant resistance to necrotrophic pathogens ${ }^{39}$. Among the WRKY target genes, EDS1 and NPR4 have been identified ${ }^{40}$. CmWRKY4 showed high expression during the early infection stage, which indicates that Chrysanthemum CmWRKY4 may act as an upstream regulator of CmEDS1, collectively contributing to the early defense response of Chrysanthemum leaves to $A$. alternata infection.

From the display of no symptoms to the expansion of necrotic lesions on Chrysanthemum leaves after $A$. alternata inoculation, ET plays a central role in defense. The expression levels of all the ET signal transduction pathway genes (CmETR1, CmCTR1, CmEIN2, and $C m E R F 2$ ) were upregulated during this transition. WGCNA also demonstrated that another major transcription factor involved in $\mathrm{Ca}^{2+}$ signaling, CmWRKY33, has a strong regulatory relationship with CmERF2.
CmWRKY33 and CmERF2 were highly expressed in the stage of necrotic lesion formation at inoculation sites and may act as important regulators in response to A. alternata infection. $\mathrm{Ca}^{2+}$ participates in different types of cellular processes as a general secondary messenger in plants, with important roles of sensing a series of extracellular stimuli and responding quickly ${ }^{28}$. We also identified the gene encoding the calcium-binding EF-hand protein $C m C M L 45$, which is involved in the $\mathrm{Ca}^{2+}$ signaling pathway. In Arabidopsis, WRKY33 is a significant regulator involved in the transcriptional regulation of metabolic and hormone responses to Botrytis cinerea infection ${ }^{41}$. Compared with that which occurred in the resistant wild type, SA levels were elevated and JAassociated responses were downregulated in susceptible WRKY33 mutants after infection with $B$. cinerea $^{42}$. As reported, ERF2 appears to be positively controlled by WRKY $33^{43}$. Both ChiB and PDF1.2 can be activated by $E R F 2$, which has been shown to lead to resistance to a number of pathogens ${ }^{44,45}$. In addition, $A C S 2$, together with $A C S 6$, is strongly correlated with the induction of $\mathrm{ET}$, and WRKY33 can bind to the W-box region in the promoters of ACS2 and ACS6, the process of which is directly associated with MPK-induced expression of ACS2 and $A C S 6^{46}$. However, whether CmWRKY33 is involved in ET signal transduction is still unknown. Our WGCNA showed that CmCML45 and CmWRKY33 have regulatory relationships with $C m E R F 2$, which was upregulated at 6 or $12 \mathrm{~h}$ after inoculation. ERF2 is an important TF for the ET signal transduction pathway. ACC synthases (ACSs) convert S-adenosyl methionine to ACC, which is the direct precursor of $\mathrm{ET}^{47}$. The results of our study also showed that two ACS homologous genes (DN61354c0_g1 and DN93750c1_g1) were upregulated at the early stage of A. alternata infection. $\mathrm{Ca}^{2+}$ influx depends on plasma membrane-localized CNGC family proteins, which are involved in the uptake of $\mathrm{Ca}^{2+}$ and initiate immune signaling in plant cells ${ }^{31,48}$. Two CNGC homologs (DN96030c0_g1 and DN93811c1_g2) were also upregulated in the early stage. The above results show that $\mathrm{Ca}^{2+}$ signal transduction and the ET pathway play important roles in the early and middle stages of A. alternata infection in Chrysanthemum leaves.

In the late stage of A. alternata infection, necrotic lesions of Chrysanthemum leaves expanded, and a brown module was identified. However, no defense genes were identified among the hub genes. Interestingly, CmDTX30 and CmDTX32, belonging to the MATE family, were identified hub genes in the brown module. Members of the MATE gene family play predominant roles in exporting toxins and other substrates ${ }^{49,50}$, and MATE proteins involved in the response to abiotic stress have been reported ${ }^{51,52}$. For example, AtDTX50, belonging to the MATE family, enhances drought tolerance in 


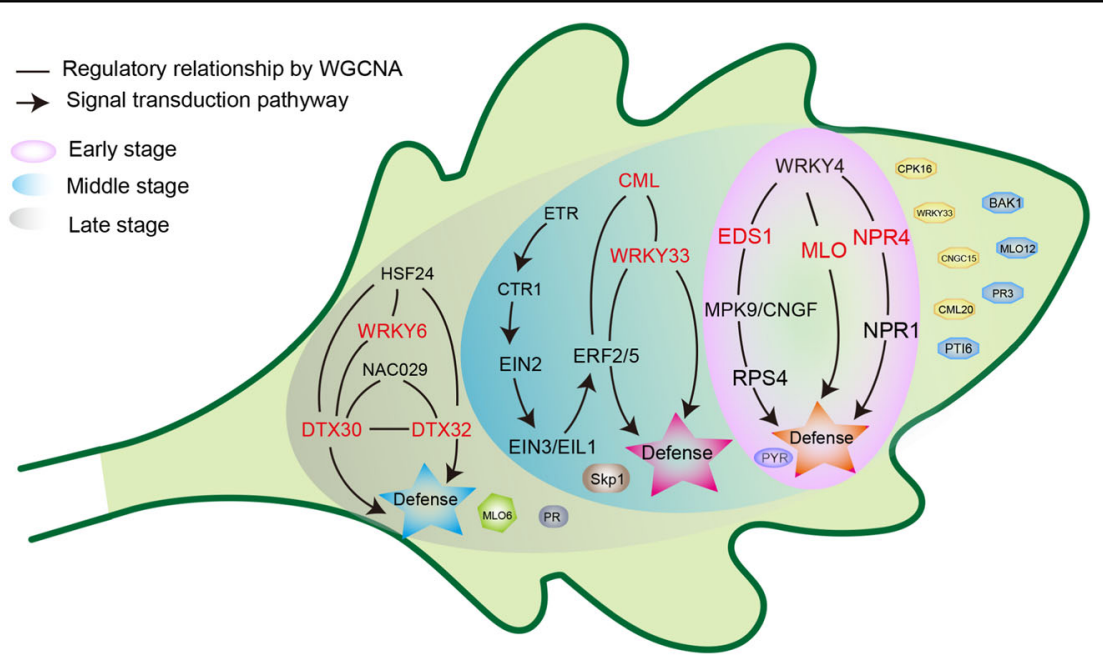

Fig. 7 Gene expression model of the response of Chrysanthemum leaves to A. alternata infection. Different background colors represent the response of Chrysanthemum leaves to $A$. alternata infection at different stages, and the gene regulatory networks at different stages are shown in different color backgrounds. The correspondence between the background color and the infection stage is shown in the figure legend. Genes responding specifically to A. alternata infection in the resistant cultivar 'Jinba' are displayed in the green background of the leaf

Arabidopsis thaliana ${ }^{53}$. However, the roles that members of the MATE gene family play in response to biotic stress remain unknown. In the brown module, CmDTX30 and CmDTX32 were highly upregulated at 24 or $36 \mathrm{~h}$ after inoculation, which indicated that some MATE family genes respond to A. alternata infection in the late stage. Alternaria alternata is a filamentous necrotrophic fungus that extracts nutrients from dead cells and produces different host-selective toxins that damage plant tissues ${ }^{54,55}$; seven pathogenic variants of $A$. alternata have been identified, which can cause disease in a variety of plants by producing host-specific toxins ${ }^{2}$. Based on the above studies, we speculate that Chrysanthemum leaves may transport toxins out of cells through multidrug resistance proteins, thereby reducing damage. Based on our results, we speculate that Chrysanthemum defends itself against A. alternata infection by reducing host-selective toxins via MATE family proteins. Additionally, ET signal transduction pathway genes exhibited peak upregulation at $24 \mathrm{hpi}$ or $36 \mathrm{hpi}$, which indicated the important roles of ET in the late stage of the Chrysanthemum response to A. alternata infection.

Furthermore, 10 differentially expressed TFs were identified from the turquoise module, indicating that these proteins may play significant roles in the resistant cultivar 'Jinba'. Among the TFs expressed in the 'Jinba' cultivar, a PTI6 homolog (CmPTI6; DN80856c2_g1) was highly expressed in the resistant cultivar 'Jinba' and differentially expressed after inoculation. PTI6, an important ERF transcription factor, specifically interacts with Pto kinase and binds to the GCC-box cis element, which is an important regulator in mediating the expression of pathogenesis-related (PR) genes ${ }^{56}$. We investigated the expression of PR homologous genes in Chrysanthemum, and the results showed that PR3-like genes (DN75226c1_g1, DN87821c0_g1, and DN88995c0_g2) were upregulated in 'Jinba' after A. alternata inoculation, with higher expression in 'Jinba' than in 'Zaoyihong' at most of the times assessed (Table S3). The results indicated that the PTI6 homolog and PR3-like genes play important roles in the response to A. alternata infection in 'Jinba'.

Collectively, we mapped a model to elucidate the response of Chrysanthemum leaves to $A$. alternata infection at different stages and compared the model of the resistant cultivar 'Jinba' to that of the susceptible cultivar 'Zaoyihong' (Fig. 7). In the early stage, the calcium signal transduction pathway was activated in response to infection. With the transmission of calcium signals, more plant immune responses were activated, especially those involving the ET signaling pathway. Because A. alternata can produce a large number of toxins, we speculate that Chrysanthemum leaves may resist damage through MDR protein detoxification. In addition, we identified a series of hub genes including PTI6, and PR3-like genes, that were highly expressed specifically in response to A. alternata infection in the resistant cultivar 'Jinba'. Our study systematically identified the immunity map of Chrysanthemum in response to A. alternata infection, revealed the defense strategies of Chrysanthemum in different infection periods, and added to our understanding of the plant immune response. The identification of the hub genes of Chrysanthemum lays a foundation for elucidating the mechanism of plant responses to pathogen infection at 
different periods. The hub genes identified from the resistant cultivar 'Jinba' provide a theoretical basis for breeding cultivars resistant to CBS.

\section{Methods}

Plant materials and $A$. alternata inoculation

Two Chrysanthemum cultivars ('Jinba' and 'Zaoyihong') and $A$. alternata were obtained from the Chrysanthemum Germplasm Resource Preserving Centre (Nanjing Agricultural University, China). Rooted seedlings were cultured in a 2:1 mixture of vermiculite and limestone. The seedlings were grown under a 16 -h photoperiod with a day/night temperature of $25^{\circ} \mathrm{C}$ and $18{ }^{\circ} \mathrm{C}$, respectively, and the relative humidity was maintained at $\sim 70 \%{ }^{57}$. Mycelia of $A$. alternata were cultured in liquid media. Mycelia collected from $1 \mathrm{~mL}$ of liquid media were placed on a Chrysanthemum leaf. After inoculation, the Chrysanthemum seedlings were grown in the dark for $36 \mathrm{~h}$. Chrysanthemum leaves were subsequently sampled at 1 , $6,12,24$, and $36 \mathrm{~h}$ post inoculation. Uninoculated leaves of Chrysanthemum were used as the control $(0 \mathrm{~h})$, which were collected at the beginning of the experiment before inoculation. Leaves infected by A. alternata collected at defined time points were used for RNA sequencing, with three replicates for each treatment.

\section{RNA extraction and sequencing}

The total RNA from 36 samples was extracted according to previously reported methods ${ }^{57}$. All 36 libraries were constructed and sequenced using the Illumina platform at Beijing Novogene (Tianjin, China) to generate 150nucleotide-long paired-end sequence reads. Clean reads were obtained from the raw reads by removing adapter sequences and low-quality reads. Chrysanthemum unigenes were acquired using Trinity (v2.4.0) for de novo assembly of the transcriptome ${ }^{58}$. The clean reads were then matched to the Chrysanthemum unigenes using RSEM software ${ }^{59}$. The frequency of individual reads was normalized to $\mathrm{FPKM}^{60}$.

\section{DEG identification and GO enrichment analysis}

DESeq was used for DEG analysis ${ }^{61}$. The padj (adjusted $P$-value) provides a criterion to determine the $P$-value threshold in multiple tests and analyses by manipulating the adjusted $P$-value. In our study, differential expression was considered when the absolute value of the $\log 2$ induction ratios of the treated samples compared with the mock treatment or control samples $(0 \mathrm{~h})$ was $\geq 1.0$ and when padj $<0.05$. To obtain comprehensive information on the function of the unigenes, seven major databases, including the nonredundant, nucleotide, Pfam, COG, Swiss-Prot, GO, and KEGG databases, were used for gene annotations. GO enrichment analysis of the DEG sets was performed using $\mathrm{BINGO}^{62}$. The $P$-value providing a criterion to determine enrichment for each represented GO term was calculated and corrected by the Benjamini and Hochberg false discovery rate (FDR). GO terms that showed corrected $P$ values $<0.05$ were considered significantly enriched. Pathway enrichment analysis was then performed to identify the series of genes involved in the response of 'Jinba' and 'Zaoyihong' to A. alternata infection using MapMan (version 3.5.1) ${ }^{63}$.

\section{Coexpression network analysis for the construction of modules}

The R package for WGCNA was used for coexpression network analysis to evaluate modules in which the genes showed high correlations ${ }^{64}$. An adjacency matrix was constructed based on normalized FPKM values. To identify modules related to phenotypic traits in the reconstruction network, the adjacency matrix was further converted to a topological overlap matrix (TOM) using the WGCNA package. After building the network, transcripts with similar expression patterns were classified into one module, and eigengenes (also called hub/key genes) for these modules were calculated. The TOM and degree were subsequently calculated; the top 20 genes according to degree were considered hub genes. Additionally, TOM $>0.3$ was considered highly correlated and strongly regulatory. Coexpression networks were visualized using Cytoscape software ${ }^{65}$.

\section{qPCR validation}

Ten genes from different modules were selected for quantitative real-time polymerase chain reaction (qPCR) analysis. Gene-specific primer pairs of the selected genes were designed using the primer design website (https://www.yeastgenome.org/primer3) (Table S2), and qPCR assays were performed as previously described ${ }^{66}$. The Chrysanthemum EF1 $\alpha$ gene was used as a reference. Transcript abundances are given as the means \pm SEs of three replicates. Relative transcription levels were calculated by using the $2^{-\Delta \Delta C T}$ method $^{67}$. Three biological replicates were performed for each sample.

\section{Chrysanthemum CmWRKY33.1 genetic transformation}

Chrysanthemum plants were transformed with Agrobacterium tumefaciens strain EHA105 according to previously reported methods ${ }^{68}$. The coding region of CmWRKY33.1 was cloned by polymerase chain reaction (PCR). According to the multiple cloning sites within the Chrysanthemum genetic transformation expression vector pENTR $^{\mathrm{TM}} 1 \mathrm{~A}$, two restriction endonuclease sites (SalI and NotI) were selected for vector construction. CmWRKY33.1 was inserted into pMDC43 by homologous recombination. The resultant pMDC43-CmWRKY33.1 vector was then used for genetic transformation, and hygromycin was used to select stable transformants. DNA and RNA were isolated from the wild-type and transformant lines, which were used 
in PCR and qPCR experiments with constructed primers (Table S2) to identify the successfully transformed lines containing CmWRKY33.1.

\section{Acknowledgements}

This work was financially supported by the National Natural Science Foundation of China (31700620), the Natural Science Fund of Jiangsu Province (BK20170722), and the Fundamental Research Funds for the Central Universities (KUN201812).

\section{Author contributions}

Y.L. and F.C. designed the experiment. Y.L. and J.X. performed the experiment. Y.L. analyzed the data and wrote the manuscript, and F.C. revised the manuscript. All authors read and approved the final manuscript.

\section{Conflict of interest}

The authors declare that they have no conflict of interest.

Supplementary Information accompanies this paper at (https://doi.org/ 10.1038/s41438-020-0245-0)

Received: 19 July 2019 Revised: 24 December 2019 Accepted: 4 January 2020

Published online: 01 March 2020

\section{References}

1. Thomma, B. P. Alternaria spp: from general saprophyte to specific parasite. Mol. Plant Pathol. 4, 225-236 (2003).

2. Takaoka, S. et al. Complex regulation of secondary metabolism controlling pathogenicity in the phytopathogenic fungus Alternaria alternata. N. Phytol. 202, 1297-1309 (2014).

3. Jones, J. D. \& Dangl, J. L. The plant immune system. Nature 444, 323-329 (2006).

4. Zipfel, C. Early molecular events in PAMP-triggered immunity. Curr. Opin. Plant Biol. 12, 414-420 (2009).

5. Tsuda, K. \& Katagiri, F. Comparing signaling mechanisms engaged in patterntriggered and effector-triggered immunity. Curr. Opin. Plant Biol. 13, 459-465 (2010).

6. Li, B., Meng, X., Shan, L. \& He, P. Transcriptional regulation of pattern-triggered immunity in plants. Cell Host Microbe 19, 641-650 (2016).

7. Nimchuk, Z., Eulgem, T., Holt, B. F. 3rd \& Dangl, J. L. Recognition and response in the plant immune system. Annu. Rev. Genet. 37, 579-609 (2003).

8. Collier, S. M. \& Moffett, P. NB-LRRs work a "bait and switch" on pathogens. Trends Plant Sci. 14, 521-529 (2009)

9. Le Roux, C. et al. A receptor pair with an integrated decoy converts pathogen disabling of transcription factors to immunity. Cell 161, 1074-1088 (2015).

10. Vlot, A. C., Dempsey, D. A. \& Klessig, D. F. Salicylic acid, a multifaceted hormone to combat disease. Annu. Rev. Phytopathol. 47, 177-206 (2009).

11. Baggs, E., Dagdas, G. \& Krasileva, K. V. NLR diversity, helpers and integrated domains: making sense of the NLR identity. Curr. Opin. Plant Biol. 38, 59-67 (2017).

12. Zipfel, C. et al. Bacterial disease resistance in Arabidopsis through flagellin perception. Nature 428, 764-767 (2004).

13. Wang, L. et al. Arabidopsis CaM binding protein CBP60g contributes to MAMPinduced SA accumulation and is involved in disease resistance against Pseudomonas syringae. PLoS Pathog. 5, e1000301 (2009).

14. van Loon, L. C., Geraats, B. P. \& Linthorst, H. J. Ethylene as a modulator of disease resistance in plants. Trends Plant Sci. 11, 184-191 (2006).

15. Zhang, L. \& Xing, D. Methyl jasmonate induces production of reactive oxygen species and alterations in mitochondrial dynamics that precede photosynthetic dysfunction and subsequent cell death. Plant Cell Physiol. 49, 1092-1111 (2008).

16. Broekgaarden, C., Caarls, L., Vos, I. A., Pieterse, C. M. \& Van Wees, S. C. Ethylene: traffic controller on hormonal crossroads to defense. Plant Physiol. 169, 2371-2379 (2015).

17. Luo, J. et al. Integrated transcriptome analysis reveals plant hormones jasmonic acid and salicylic acid coordinate growth and defense responses upon fungal infection in poplar. Biomolecules 9, https://doi.org/10.3390/ biom9010012 (2019).

18. Adie, B. A. et al. ABA is an essential signal for plant resistance to pathogens affecting JA biosynthesis and the activation of defenses in Arabidopsis. Plant Cell 19, 1665-1681 (2007).

19. Ton, J., Flors, V. \& Mauch-Mani, B. The multifaceted role of ABA in disease resistance. Trends Plant Sci. 14, 310-317 (2009).

20. Cai, S. et al. Evolutionary conservation of ABA signaling for stomatal closure. Plant Physiol. 174, 732-747 (2017).

21. Ohme-Takagi, M., Suzuki, K. \& Shinshi, H. Regulation of ethylene-induced transcription of defense genes. Plant Cell Physiol. 41, 1187-1192 (2000).

22. Singh, K. Foley, R. C. \& Onate-Sanchez, L. Transcription factors in plant defense and stress responses. Curr. Opin. Plant Biol. 5, 430-436 (2002).

23. Dolmetsch, R. E., Xu, K. \& Lewis, R. S. Calcium oscillations increase the efficiency and specificity of gene expression. Nature 392, 933-936 (1998).

24. Poovaiah, B. W., Du, L., Wang, H. \& Yang, T. Recent advances in calcium/ calmodulin-mediated signaling with an emphasis on plant-microbe interactions. Plant Physiol. 163, 531-542 (2013).

25. Gust, A. A. \& Felix, G. Receptor like proteins associate with SOBIR1-type of adaptors to form bimolecular receptor kinases. Curr. Opin. Plant Biol. 21, 104-111 (2014).

26. Yuan, P., Jauregui, E., Du, L., Tanaka, K. \& Poovaiah, B. W. Calcium signatures and signaling events orchestrate plant-microbe interactions. Curr. Opin. Plant Biol. 38, 173-183 (2017)

27. Zipfel, C. \& Oldroyd, G. E. Plant signalling in symbiosis and immunity. Nature 543, 328-336 (2017)

28. Edel, K. H., Marchadier, E., Brownlee, C., Kudla, J. \& Hetherington, A. M. The evolution of calcium-based signalling in plants. Curr. Biol. 27, R667-R679 (2017).

29. Brandt, B. et al. Calcium specificity signaling mechanisms in abscisic acid signal transduction in Arabidopsis guard cells. Elife 4, https://doi.org/10.7554/ elife.03599 (2015)

30. Raz, V. \& Fluhr, R. Calcium requirement for ethylene-dependent responses. Plant cell 4, 1123-1130 (1992).

31. Reddy, A. S., Ali, G. S., Celesnik, H. \& Day, I. S. Coping with stresses: roles of calcium- and calcium/calmodulin-regulated gene expression. Plant Cell 23, 2010-2032 (2011).

32. Zhao, N. et al. Comparative Transcriptome analysis of waterlogging-sensitive and waterlogging-tolerant Chrysanthemum morifolium cultivars under waterlogging stress and reoxygenation conditions. Int. J. Mol. Sci. 19, https://doi.org/ 10.3390/ijms19051455 (2018).

33. Wang, H., Lin, J., Chang, Y. \& Jiang, C. Z. Comparative transcriptomic analysis reveals that ethylene/H2O2-mediated hypersensitive response and programmed cell death determine the compatible interaction of sand pear and Alternaria alternata. Front. Plant Sci. 8, 195 (2017)

34. Wirthmueller, L., Zhang, Y., Jones, J. D. \& Parker, J. E. Nuclear accumulation of the Arabidopsis immune receptor RPS4 is necessary for triggering EDS1dependent defense. Curr. Biol. 17, 2023-2029 (2007).

35. Heidrich, K. et al. Arabidopsis EDS1 connects pathogen effector recognition to cell compartment-specific immune responses. Science 334,1401-1404 (2011).

36. Huang, P. Y., Catinot, J. \& Zimmerli, L. Ethylene response factors in Arabidopsis immunity. J. Exp. Bot. 67, 1231-1241 (2016).

37. Tsuda, K., Sato, M., Stoddard, T., Glazebrook, J. \& Katagiri, F. Network properties of robust immunity in plants. PLoS Genet. 5, e1000772 (2009).

38. Cui, $\mathrm{H}$. et al. Antagonism of transcription factor MYC2 by EDS1/PAD4 complexes bolsters salicylic acid defense in Arabidopsis effector-triggered immunity. Mol. Plant 11, 1053-1066 (2018).

39. Lai, Z., Vinod, K., Zheng, Z., Fan, B. \& Chen, Z. Roles of Arabidopsis WRKY3 and WRKY4 transcription factors in plant responses to pathogens. BMC Plant Biol. $\mathbf{8}$ 68 (2008).

40. Birkenbihl, R. P., Kracher, B., Roccaro, M. \& Somssich, I. E. Induced genome-wide binding of three Arabidopsis WRKY transcription factors during early MAMPtriggered immunity. Plant Cell 29, 20-38 (2017).

41. Liu, S., Kracher, B., Ziegler, J., Birkenbihl, R. P. \& Somssich, I. E. Negative regulation of ABA signaling by WRKY33 is critical for Arabidopsis immunity towards Botrytis cinerea 2100. Elife 4, e07295 (2015).

42. Liu, S., Ziegler, J., Zeier, J., Birkenbihl, R. P. \& Somssich, I. E. Botrytis cinerea B05.10 promotes disease development in Arabidopsis by suppressing WRKY33-mediated host immunity. Plant Cell Environ. 40, 2189-2206 (2017). 
43. Birkenbihl, R. P., Diezel, C. \& Somssich, I. E. Arabidopsis WRKY33 is a key transcriptional regulator of hormonal and metabolic responses toward Botrytis cinerea infection. Plant Physiol. 159, 266-285 (2012).

44. Brown, R. L., Kazan, K., McGrath, K. C., Maclean, D. J. \& Manners, J. M. A role for the GCC-box in jasmonate-mediated activation of the PDF1.2 gene of Arabidopsis. Plant Physiol. 132, 1020-1032 (2003).

45. Meng, X. et al. Phosphorylation of an ERF transcription factor by Arabidopsis MPK3/MPK6 regulates plant defense gene induction and fungal resistance. Plant Cell 25, 1126-1142 (2013)

46. Li, G. et al. Dual-level regulation of ACC synthase activity by MPK3/MPK6 cascade and its downstream WRKY transcription factor during ethylene induction in Arabidopsis. PLoS Genet. 8, e1002767 (2012).

47. Dubois, M., Van den Broeck, L. \& Inze, D. The pivotal role of ethylene in plant growth. Trends Plant Sci. 23, 311-323 (2018).

48. Ma, W. et al. Ca2+, CAMP, and transduction of non-self perception during plant immune responses. Proc. Natl Acad. Sci. USA 106, 20995-21000 (2009).

49. He, X. et al. Structure of a cation-bound multidrug and toxic compound extrusion transporter. Nature 467, 991-994 (2010).

50. Lu, P. et al. Genome-wide analysis of multidrug and toxic compound extrusion (MATE) family in Gossypium raimondii and Gossypium arboreum and its expression analysis under salt, cadmium, and drought stress. G3 8, 2483-2500 (2018).

51. Liu, J., Li, Y., Wang, W., Gai, J. \& Li, Y. Genome-wide analysis of MATE transporters and expression patterns of a subgroup of MATE genes in response to aluminum toxicity in soybean. BMC Genomics 17, 223 (2016).

52. Li, N. et al. Genome-wide analysis of MATE transporters and molecular characterization of aluminum resistance in Populus. J. Exp. Bot. 68, 5669-5683 (2017).

53. Zhang, H. et al. A DTX/MATE-type transporter facilitates abscisic acid efflux and modulates ABA sensitivity and drought tolerance in Arabidopsis. Mol. Plant 7 1522-1532 (2014)

54. Estiarte, N., Lawrence, C. B., Sanchis, V., Ramos, A. J. \& Crespo-Sempere, A. LaeA and VeA are involved in growth morphology, asexual development, and mycotoxin production in Alternaria alternata. Int. J. Food Microbiol. 238, 153-164 (2016).
55. Sanchez-Lopez, A. M. et al. Arabidopsis responds to Alternaria alternata volatiles by triggering plastid phosphoglucose isomerase-independent mechanisms. Plant Physiol. 172, 1989-2001 (2016).

56. Gu, Y. Q. et al. Tomato transcription factors pti4, pti5, and pti6 activate defense responses when expressed in Arabidopsis. Plant Cell 14, 817-831 (2002).

57. $\mathrm{Li}, \mathrm{H}$. et al. RNA-Seq derived identification of differential transcription in the Chrysanthemum leaf following inoculation with Alternaria tenuissima. BMC Genomics 15, 9 (2014).

58. Grabherr, M. G. et al. Full-length transcriptome assembly from RNA-Seq data without a reference genome. Nat. Biotechnol. 29, 644-652 (2011).

59. Li, B. \& Dewey, C. N. RSEM: accurate transcript quantification from RNA-Seq data with or without a reference genome. BMC Bioinform. 12, 323 (2011).

60. Trapnell, C. et al. Transcript assembly and quantification by RNA-Seq reveals unannotated transcripts and isoform switching during cell differentiation. Nat. Biotechnol. 28, 511-515 (2010).

61. Anders, S. \& Huber, W. Differential expression analysis for sequence count data. Genome Biol. 11, R106 (2010).

62. Maere, S., Heymans, K. \& Kuiper, M. BiNGO: a Cytoscape plugin to assess overrepresentation of gene ontology categories in biological networks. Bioinformatics 21, 3448-3449 (2005).

63. Thimm, O. et al. MAPMAN: a user-driven tool to display genomics data sets onto diagrams of metabolic pathways and other biological processes. Plant J. 37, 914-939 (2004).

64. Zhang, B. \& Horvath, S. A general framework for weighted gene co-expression network analysis. Stat. Appl. Genet. Mol. Biol. 4, Article17 (2005).

65. Stuart, J. M., Segal, E., Koller, D. \& Kim, S. K. A gene-coexpression network for global discovery of conserved genetic modules. Science 302, 249-255 (2003).

66. Song, A. et al. Comprehensive analysis of mitogen-activated protein kinase cascades in Chrysanthemum. PeerJ 6, e5037 (2018).

67. Livak, K. J. \& Schmittgen, T. D. Analysis of relative gene expression data using real-time quantitative PCR and the 2(-Delta Delta $C(T)$ ) Method. Methods 25 402-408 (2001).

68. Li, P. et al. The over-expression of a Chrysanthemum WRKY transcription factor enhances aphid resistance. Plant Physiol. Biochem. 95, 26-34 (2015). 